\title{
Analytical solutions for dense, inclined, granular flow over a rigid, bumpy base
}

\author{
James Jenkins ${ }^{1 *}$ and Diego Berzi ${ }^{2}$ \\ ${ }^{1}$ Cornell University, Ithaca, NY, 14850, USA \\ ${ }^{2}$ Politecnico di Milano, Milan 20133, Italy
}

\begin{abstract}
We first phrase a boundary-value problem for a dense, steady, fully-developed, gravitational flow of identical inelastic spheres over in inclined bumpy base in the absence of sidewalls. We then obtain approximate analytical solutions for the profiles of the solid volume fraction, the strength of the velocity fluctuations, and the mean velocity of the flow. We compare these with those obtained in numerical solutions of the exact equations.
\end{abstract}

\section{Introduction}

Flows of granular materials down inclines occur often in Nature and in industry. Consequently, it is important to be able to describe and predict them. Because such flow take place under the influence of gravity, they typically involve solid volume fractions greater than 0.30 . In this sense, they are dense. Classical dense flow kinetic theory (e.g., [1]) has the capability of describing such flows (e.g,, [2]), provided that the volume fraction does not exceed about 0.49 . Above this, the predictions for the properties of inclined flow fail, and alternatives must be employed.

One such alternative is a nonlocal extension [3] of the $\mu-I$ rheology $[4,5]$ that has been applied to dense, inclined flows [6]. Another is an extension of the kinetic theory that takes into account, in a phenomenological way, the existence of velocity correlations $[7,8,9]$. In fact, the two approaches have much in common $[10,11]$.

Here, we employ the latter to phrase a boundaryvalue problem for a dense, steady, fully-developed, gravitational flow of identical inelastic spheres over an inclined bumpy base in the absence of sidewalls. We then obtain approximate analytical solutions for the profiles of the solid volume fraction, the strength of the velocity fluctuations, and the mean velocity of the flow.

\section{Model}

We consider a steady, dense, fully-developed flow of identical, frictional, inelastic spheres of mass $m$, material density $\rho_{s}$, diameter $d$, with coefficient of restitution, $e$, and sliding friction, $\mu$. The flow is driven by gravity over a rigid, bumpy bed, inclined at an angle of $\phi$, in the absence of sidewalls. The bed is composed of spheres, identical to those of the flow, randomly affixed to a plane with an average distance of $\sigma$ between their edges.

The $x$-axis is in the direction of flow, the $y$-axis is normal to the flow, directed upward from the base, and $g$ is the gravitational acceleration. Primes denote derivatives with respect to $y$. The solid volume fraction is $v$, the flow velocity is $u$, and the measure of the strength of the velocity fluctuations, $T$, is one third their meansquared value. We employ extended kinetic theory to phrase a boundary-value problem for the variations of these fields across the flow and obtain approximate, analytical solutions for them that we compare to numerical solutions of the exact equations.

\subsection{Momentum balance}

The component of the momentum balance along the flow governs the variation of the shear stress $\mathrm{S}$ :

$$
S^{\prime}=-\rho_{s} v g \sin \phi
$$

While the component of the momentum balance normal to the flow governs the variation of the pressure P:

$$
P^{\prime}=-\rho_{s} v g \cos \phi .
$$

When integrated together,

$$
\frac{S}{P}=\tan \phi
$$

The flows are assumed to be so dense that $v$ is greater than 0.49 everywhere within the flow. Then, in the expressions for the stress, we retain only terms that result from collisional transfer of momentum and ignore those that result from transport. Then, [1]

* Corresponding author: jim.jenkins@cornell.edu

A video is available at https://doi.org/10.48448/dyv1-c459 


$$
P=2 \rho_{s} v(1+e) G T
$$

where

$$
G=5.69 v \frac{v_{c}-0.49}{v_{c}-v},
$$

in which the critical volume fraction, $v_{c}$, at which the collisional interactions become singular, is given by [12]

$$
v_{c}=0.58+(0.64-0.58) e^{-4.5 \mu}
$$

and

where [1]

$$
S=\frac{8 \hat{J}}{5 \pi^{1 / 2}} d \rho_{s} v(1+e) G T^{1 / 2} u^{\prime}
$$

$$
\hat{J}=\frac{1}{2}+\frac{\pi}{4} \frac{(3 e-1)(1+e)}{24-(1-e)(11-e)} .
$$

The dependance of the shear stress on the volume fraction may be eliminated in favor of a dependance on the pressure:

$$
S=\frac{4 \hat{J}}{5 \pi^{1 / 2}} d \frac{P}{T^{1 / 2}} u^{\prime}
$$

\subsection{Energy balance}

The balance of fluctuation energy in the flow relates the gradient of the energy flux, $Q$, the working of the shear stress, and the rate of collisional dissipation, $\Gamma$ :

$$
-Q^{\prime}+S u^{\prime}-\Gamma=0
$$

Upon retaining only the collisional transfers, [1]

$$
Q=-\frac{4 \hat{M}}{\pi^{1 / 2}} d \rho_{s} v(1+e) G T^{1 / 2} T^{\prime},
$$

where

$$
\hat{M}=\frac{1}{2}+\frac{9 \pi}{8} \frac{(2 e-1)(1+e)}{16-7(1-e)}
$$

or

$$
Q=-\frac{2 \hat{M}}{\pi^{1 / 2}} \frac{P d}{T^{1 / 2}} T^{\prime}
$$

and [1]

$$
\Gamma=\frac{12}{\pi^{1 / 2}}\left(1-\varepsilon^{2}\right) \frac{\rho_{s} v G T^{3 / 2}}{L}=\frac{6}{\pi^{1 / 2}}(1-\varepsilon) \frac{P T^{1 / 2}}{L},
$$

where $\varepsilon=e-(3 / 2) \mu \mathrm{e}^{-3 \mu}$ is the effective restitution [12] and $L$ is the correlation length [12]

$$
\frac{L}{d}=f \frac{d u^{\prime}}{T^{1 / 2}}=f \frac{5 \pi^{1 / 2}}{4 \hat{J}} \tan \phi,
$$

with

$$
f=\left[\frac{2 \hat{J}}{15(1-\varepsilon)}\right]^{1 / 2}\left[1+\frac{26}{15}(1-\varepsilon)\left(\frac{v-0.49}{0.64-v}\right)\right]^{3 / 2}
$$

With Eqs. (9), (13), and (14), the energy equation becomes

$$
\left(-\frac{2 \hat{M}}{\pi^{1 / 2}} \frac{P d}{T^{1 / 2}} T^{\prime}\right)^{\prime}=\frac{5 \pi^{1 / 2}}{4 \hat{J}} \frac{S^{2}}{P} \frac{T^{1 / 2}}{d}-\frac{6}{\pi^{1 / 2}}(1-\varepsilon) \frac{P T^{1 / 2}}{L}
$$

When written in terms of the fluctuation velocity, $w=T^{1 / 2}$, this is

$$
w^{\prime \prime}+\frac{P^{\prime}}{P} w^{\prime}-\frac{\pi^{1 / 2}}{4 \hat{M}}\left(\frac{6}{\pi^{1 / 2}} \frac{1-\varepsilon}{L}-\frac{5 \pi^{1 / 2}}{4 \hat{J}} \tan ^{2} \phi\right) \frac{w}{d^{2}}=0 .
$$

\subsection{Boundary conditions}

At the free surface, we assume that $v=0.49$ and make the rough approximation that $Q \propto w^{\prime}=0$. At the rigid, bumpy base, the flow momentum balance gives the slip velocity, [13]

$$
u=\left(\frac{\pi}{2}\right)^{1 / 2} F w \tan \phi
$$

where the slip coefficient, $F$, is given by

$$
F=\frac{2}{\theta^{2}}-\frac{5 \pi}{24(1+e) \hat{J}}+\frac{25+300 \sqrt{2}-7(1+e) \hat{J}}{360(1+e) \hat{J}} \theta^{2},
$$

in which $\theta$ is the bumpiness of the boundary, defined in terms of the diameter of the boundary spheres and the average separation of their edges by $\sin \theta=(d+\sigma)$ $/(2 d)$.

The energy balance at the base relates the energy flux, the rate of working of the shear stress through the slip velocity, and the rate of collisional dissipation, $D$ : [13]

where

$$
Q=S u-D,
$$




$$
D=\left(\frac{2}{\pi}\right) H \frac{(1-\varepsilon)}{L} P w
$$

with

$$
H=1+\frac{1}{4} \theta^{2}
$$

Using

$$
Q=-\frac{4 \hat{M}}{\pi^{1 / 2}} P d w^{\prime}
$$

the energy balance at the base may then be written as

$$
w^{\prime}=-\frac{\sqrt{2}}{4 \hat{M}}\left(\frac{\pi}{2} F \tan ^{2} \phi-\frac{1-\varepsilon}{\bar{L}} H\right) w \equiv-B w .
$$

\section{Approximate analytical solutions}

We make lengths dimensionless by $d$, velocities by $(g d)^{1 / 2}$, stress by $\rho_{s} g d$, and prescribe the mass holdup:

$$
M \equiv \int_{0}^{h} v(y) d y .
$$

To obtain the solution for $w$, we assume that the pressure vanishes at the top of the flow and approximate the integration of the normal momentum balance and the dimensionless pressure by

$$
P=\bar{v}(h-y) \cos \phi,
$$

where $\bar{v}$ is the depth-averaged volume fraction, and

$$
P=2 \bar{v}(1+e) 5.69 v \frac{v_{c}-0.49}{v_{c}-v} w^{2}
$$

respectively. Then

$$
\frac{P^{\prime}}{P}=-\frac{1}{h-y}
$$

Next, we replace the varying volume fraction in the expression for the correlation length by it average value $M / h$, where $h$ is to be determined. Then,

$\bar{L}=\left[\frac{2 \hat{J}}{15(1-\varepsilon)}\right]^{1 / 2}\left[1+\frac{26}{15}(1-\varepsilon)\left(\frac{M-0.49 h}{0.64 h-M}\right)\right]^{3 / 2} \frac{5 \pi^{1 / 2}}{4 \hat{J}} \tan \phi$,

With these, the Eq. (18) for $w$ becomes

$$
w^{\prime \prime}-\frac{1}{h-y} w^{\prime}-\kappa^{2} w=0
$$

where

$$
\kappa^{2} \equiv \frac{\pi^{1 / 2}}{4 \hat{M}}\left[\frac{6}{\pi^{1 / 2}} \frac{(1-\varepsilon)}{\bar{L}}-\frac{5 \pi^{1 / 2}}{4 \hat{J}} \tan ^{2} \phi\right]
$$

We introduce the dimensionless distance $s=h-y$ from the top of the flow and denote a derivative with respect to it by an overdot. Then, Eq. (31) becomes

$$
\ddot{w}+\frac{1}{S} \dot{w}-\kappa^{2} w=0
$$

Solutions to this are $w=C_{1} I_{0}(\kappa s)+C_{2} K_{0}(\kappa s)$, where $I_{0}$ and $K_{0}$ are modified Bessel functions of order zero. Because $K_{0}(0)$ is infinite, we discard it. At $s=h$, $w_{0} \equiv w(y=0)=C_{1} I_{0}(\kappa h)$ so $C_{1}=w_{0} / I_{0}(\kappa h)$ and

$$
w(y)=w_{0} \frac{I_{0}[\kappa(h-y)]}{I_{0}(\kappa h)}
$$

With Eq. (34), we consider the boundary condition (25), $w^{\prime}=-B w$. We use the identity $d I_{0}(x) / d x=I_{1}(x)$ to obtain

$$
\kappa I_{1}(\kappa h)=B I_{0}(\kappa h)
$$

We regard this as a nonliner equation for $h$ and use the Matlab function fsolve to determine $h$ as a function of $M$ and the other known parameters. Fig. (1) shows $h$ as a function of $M$ for the parameters indicated.

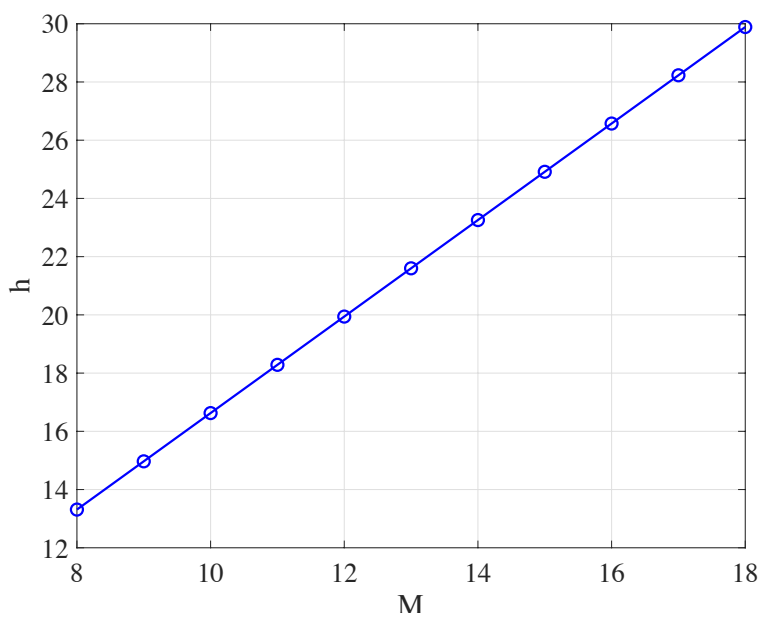

Fig. 1 Dimensionless flow height versus dimensionless mass hold-up for $e=0.80, \mu=0.10, \phi=0.29\left(16.6^{\circ}\right)$, and $\theta=\pi / 5$.

The introduction of the correlation length and its dependence on volume fraction permits the extended kinetic theory to predict the observed differences in height at a given inclination (e.g., [14]), in contrast to the single 
height at a given angle of inclination predicted by the classical kinetic theory [2].

For the determination of the average flow velocity, Eq. (31) for $S$ may first be expressed as a differential equation for $u^{\prime}$ :

$$
u^{\prime}=\frac{5 \pi^{1 / 2}}{4 \hat{J}} \tan \phi w
$$

With Eq. (34), this may be integrated to

$$
\begin{aligned}
& u(y)=\left(\frac{\pi}{2}\right)^{1 / 2} w_{0} \tan \phi\left\{F+\frac{5}{2 \sqrt{2} \hat{J}} \frac{1}{I_{0}(\kappa h)}\right. \\
& \left.\times\left[h_{1} F_{2}\left(\frac{1}{2} ; 1, \frac{3}{2} ; \frac{\kappa^{2} h^{2}}{4}\right)-(h-y)_{1} F_{2}\left(\frac{1}{2} ; 1, \frac{3}{2} ; \frac{\kappa^{2}(h-y)^{2}}{4}\right)\right]\right\},
\end{aligned}
$$

where ${ }_{1} F_{2}$ is the generalized hypergeometric function with indicated arguments, evaluated in Matlab using hypergeom $\left(1 / 2,[1,3 / 2], \kappa^{2}(h-y)^{2} / 4\right)$, for example.

Finally, an approximate determination of $v(y)$ may be obtained by giving up the assumption that the pressure at the top of the flow, $P_{h}$, is not zero and choosing its value so that $v=0.49$ there. Then, upon replacing $v$ by its depth-averaged value $\bar{v}$ in the dimensionless form of Eq. (2) and integrating,

$$
P(y)-P_{0}=-\cos \phi \int_{0}^{y} v(\xi) d \xi \doteq-\bar{v} y \cos \phi
$$

where $P_{0}=P_{h}+\bar{v} h$. Upon using Eqs (27) and (28) in this,

$$
2(1+e) 5.69 v \frac{v_{c}-0.49}{v_{c}-v} w^{2} \doteq \frac{P_{h}}{\bar{v}}+(h-y) \cos \phi ;
$$

so,

$$
\begin{aligned}
& v(y)=\left[P_{h} / \bar{v}+(h-y) \cos \phi\right] v_{c} \\
& \quad /\left[2(1+e) 5.69\left(v_{c}-0.49\right) w^{2}(y)+P_{h} / \bar{v}+(h-y) \cos \phi\right]
\end{aligned}
$$

Then, if $v(h)=0.49$,

$$
P_{h}=2(1+e) 5.69 w_{h}^{2}(0.49) \bar{v},
$$

with $w_{h}^{2}=w_{0}^{2} /\left[I_{0}(\kappa h)\right]^{2}$

The expressions for the profiles of $w, u$, and $v$ are all parameterized in terms of $w_{0}$. To obtain an estimate of it, we evaluate Eq. (39) at $y=0$, using Eq. (41):

$$
2(1+e) 5.69\left\{v_{0} \frac{v_{c}-0.49}{v_{c}-v_{0}}-\frac{0.49}{\left[I_{0}(\kappa h)\right]^{2}}\right\} w_{0}^{2}=h \cos \phi
$$

In this, we make the rough approximation that $v_{0}=\bar{v}$ :

$$
\left\{\frac{M}{h} \frac{v_{c}-0.49}{v_{c}-M / h}-\frac{0.49}{\left[I_{0}(\kappa h)\right]^{2}}\right\} w_{0}^{2}=\frac{h \cos \phi}{2(1+e) 5.69} .
$$

This determination of $w_{0}$ completes the solutions.

\section{Comparisons and conclusions}

In Fig. 1, we show profiles predicted for $v, w$, and $u$ by the aproximate analytical solutions and by the numerical integration of the system of differential equations and boundary conditions using the Matlab code $b v p 4 c$. The agreement is generally good, with the greatest error at the rigid base. This error is associated with the approximation made to determine $w o$.
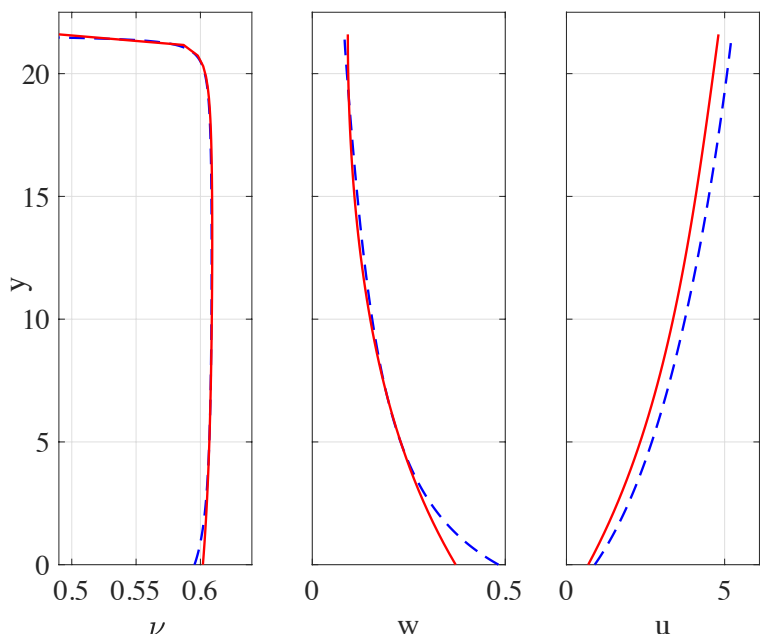

Fig. 2 Dimensionless flow height versus volume fraction, $v$ dimensionless fluctuation velocity, $w$, and mean velocity, $u$, for $M=13$ and the parameter values of Fig. 1. The dashed curves are the numerical integrations of the differential equation; the solid curves are the approximate analytical solutions.

These results are encouraging and support an attempt to improve upon them.

\section{References}

[1] V. Garzó, J.W. Dufty, Phys. Rev. E 59, 5895 (1999)

[2] J.T. Jenkins, Appl. Mech. Rev. 47, S240 (1994)

[3] GDR MiDi, Eur. Phys. J. E 14, 341 (2004)

[4] K. Kamrin, G. Koval, Phys. Rev. Lett. 108, 178301 (2012)

[5] D.L. Henann, K. Kamrin, Proc. Natl. Acad. Sci. USA 110, $6730(2013)$

[6] K. Kamrin and D.L. Henann, Soft Matter 11, 179 (2015)

[7] J.T. Jenkins, Gran. Matt.10, 47 (2007).

[8] J.T. Jenkins, D. Berzi, Gran. Matt. 12, 151 (2010)

[9] D. Gollin, D. Berzi, E.T. Bowman, Gran. Matt. 19 (2017)

[10] Q. Zhang, K. Kamrin, Phys. Rev. Lett. 118, 058001 (2017)

[11] J.T. Jenkins, D. Berzi, Soft Matter 11, 4799 (2015)

[12] D. Berzi, D. Vescovi, Phys. Fluids 27, 013302 (2015)

[13] M.W. Richman, Mech. Matls. 16, 211 (1993)

[14] L.E. Silbert, D. Ertas, G. S. Grest, T. C. Halsey, D. Levine, S. J. Plimpton, Phys. Rev. E 64, 051302 (2001) 